\title{
Molecular Characterization and Screening for Fusarium (Fusarium solani) Resistance in Chilli (Capsicum annuum L.) Genotypes
}

\author{
B.V. Tembhurne ${ }^{1}$, B. Belabadevi ${ }^{1}$, B. Kisan ${ }^{2}$, I.S. Tilak ${ }^{2}$, \\ D.S. Ashwathanarayana ${ }^{3}$, Suvarna ${ }^{1}$, Nidagundi ${ }^{1}$ and M.K. Naik ${ }^{3}$ \\ ${ }^{1}$ Department of Genetics and Plant Breeding, ${ }^{2}$ Department of Molecular Biology and \\ Agricultural Biotechnology, ${ }^{3}$ Department of Plant Pathology, College of Agriculture, University \\ of Agricultural Sciences, Raichur-584104, Karnataka, India \\ *Corresponding author
}

\begin{tabular}{|c|c|}
\hline \multicolumn{2}{|r|}{ A B S T R A C T } \\
\hline & \multirow{5}{*}{$\begin{array}{l}\text { Chilli (Capsicum annuum L.) a spice cum vegetable crop belongs to the family } \\
\text { Solanaceae. It is an indispensable spice essentially used in every Indian cuisine due to its } \\
\text { pungency, taste, appealing odour and flavour. Chilli fruits are rich sources of vitamin A, C, } \\
\text { E and P. The present study was undertaken during kharif } 2014 \text { at experimental farm, } \\
\text { Department of Genetics and Plant Breeding, College of Agriculture, Raichur during kharif } \\
2014 \text { to screen genotypes resistant to Fusarium wilt and molecular characterization for } \\
\text { Fusarium wilt resistance in } \mathrm{M}_{2} \text { and } \mathrm{M}_{3} \text { generation. Among } 62 \text { selected genotypes } 26 \\
\text { genotypes showed resistant, } 32 \text { genotypes showed moderately resistant and } 3 \text { genotypes } \\
\text { registered susceptible reaction in } \mathrm{M}_{2} \text { generation. However, only one genotype recorded } \\
\text { highly susceptible reaction to Fusarium wilt. In } \mathrm{M}_{3} \text { generation among } 73 \text { screened } \\
\text { genotypes } 32 \text { showed resistant, } 29 \text { genotypes were found to be moderately resistant and } 12 \\
\text { genotypes exhibited susceptible reaction. Molecular diversity assessment in } \mathrm{M}_{3} \text { chilli } \\
\text { mutant genotypes using RAPD markers revealed high variability among the genotypes at } \\
\text { molecular level and high divergence among these genotypes. }\end{array}$} \\
\hline Keywords & \\
\hline $\begin{array}{l}\text { Capsicum, Chilli, } \\
\text { Fusarium wilt, } \\
\text { M2 and M3 } \\
\text { generation, RAPD. }\end{array}$ & \\
\hline Article Info & \\
\hline $\begin{array}{l}\text { Accepted: } \\
\text { 19 July } 2017 \\
\text { Available Online: } \\
\text { 10 September } 2017\end{array}$ & \\
\hline
\end{tabular}

\section{Introduction}

The chilli crop suffers from many diseases among these wilt, anthracnose, murda complex, leaf spot, and powdery mildew are major problems. Wilt caused by Fusarium solani is the principal disease of chilli and has assumed a serious problem during the last few years and is causing a great concern to vegetable growers. Average losses due to this disease are up to 30 to 40 per cent and in some fields it was so serious that every plant was killed by wilt before first picking. It was more serious in chilli growing tracts of India Singh et al., 1998 and also in Karnataka particularly in black cotton soil results up to 25 per cent yield loss (Madhukar and Naik, 2004). The incidence of wilt varied from 0 to 75 per cent in different states of India (Anon., 2005). Host plant resistance has been a choice in all crop improvement programmes and is perhaps the best method available to tackle the soil borne diseases in particular. Fusarium wilt is a typical soil borne disease which can be mitigated appropriately by use of disease resistant cultivars. Further the use of resistant variety will go a long way not only in reducing loss due to disease but also in 
avoiding fungicidal toxicity likely to occur due to their application to soil, and some of the morphological characters helps in yield improvement. Mutation breeding often used to correct defects in cultivar which has a set of good agronomic characteristics (Sigurbjornson, 1977). Chemical mutagens such as Ethyl Methane Sulphonate (EMS) appear to induce higher proportions of point mutations than chromosomal aberrations (Konzak et al., 1977). Different types of morphological mutants were isolated through chemical mutagenesis in Capsicum annuum L. by various workers (Subhash et al., 1981; Raghuvanshi and Singh, 1982; Rostaino, 1983). Hence the present study was undertaken to screen the genotypes and molecular characterization foe Fusarium wilt in chilli.

\section{Materials and Methods}

Seeds of Fusarium wilt resistant genotype i.e., P3 was treated using 3 different doses of Ethyl Methyl Sulphonate (EMS) with concentration of $0.5 \%, 1.0 \%$ and $1.5 \%$ with control. The experimental material study consists of selfed seeds of 62 plants of $\mathrm{M}_{1}$ generation (29 genotypes of $0.5 \%$ dose, 21 genotypes of $1.0 \%$ dose and 12 genotypes of $1.5 \%$ dose), 73 plants of $\mathrm{M}_{2}$ generation (33 genotypes of $0.5 \%$ dose, 26 genotypes of $1.0 \%$ dose and 14 genotypes of $1.5 \%$ dose) along with genotype P3 as resistant and Byadagi dabbi as susceptible check. The $\mathrm{M}_{1}$ population was planted in Kharif 2013-2014 to produce $\mathrm{M}_{2}$ population. The $\mathrm{M}_{2}$ and $\mathrm{M}_{3}$ population was sown in order to study the resistant to Fusarium wilt during Kharif 2014-2015.

\section{Isolation of $F$. solani f. sp. Capsici}

The causal pathogen was isolated from the diseased root samples of chilli, after surface sterilization and were aseptically transferred to Potato Dextrose Agar (PDA) medium in Petri-plates and incubated at $25 \pm 2^{\circ} \mathrm{C}$ for 7 days. The isolates were purified by single spore and hypal tip isolation methods. The pathogen was identified with the help of description, and their morphological/cultural characteristics.

\section{Mass multiplication of Fusarium on PDA}

The fungus was mass multiplied on Potato Dextrose Broth (PDB). $100 \mathrm{ml}$ of PDB was taken into $250 \mathrm{ml}$ conical flask and autoclaved at $1.1 \mathrm{~kg} / \mathrm{cm}^{2}$ for 20 minutes. The mycelial disc cut from the margin of a week old culture grown on Petri dish was inoculated into PDB under aseptic conditions. The flasks were incubated at $28 \pm 10 \mathrm{C}$ for 15 days. The mycelium mats were collected after 15 days by filtering with Whatsman No 42 filter paper disc of $12.5 \mathrm{~cm}$ diameter and washed with sterile water. The spore suspension was prepared using waring blender to disturb the spores in sterile water and filtered through cheese-cloth before use and spore load $\mathrm{ml}^{-1}$ was computed by using a Haemocytometer.

\section{Pathogenicity test by rapid root dip transplanting technique}

Rapid root dip transplanting technique method developed by Naik et al., (1996) was used to screen the genotypes. Chilli seedlings were raised in a plastic trays containing sterilized sand in a nylon net house and protected with two insecticidal sprays of Malathion $(0.1 \%)$ and Monocrotophos $(0.05 \%)$ to prevent the viral disease. Three weeks old seedlings were removed, roots thoroughly washed in running tap water and $3 \mathrm{~mm}$ tip of roots were cut and immersed in spore suspension of $F$. solani and planted in a plastic bags containing sterilized soil. Then plants were planted in plastic bags and maintained. In cases where isolates produced 
typical wilting symptoms, the fungus was successfully re-isolated and Koch's postulates proved.

\section{Computation of disease index}

The plant survival data as on 30 DAT was recorded to ascertain the Fusarium wilt disease. Plant survival (\%) was calculated as:

Plant survival $\%=$

Number of healthy plants in the last recording x 100

Number of plants established

PDI \% = - Number of plants wilted

Total number of plants observed

The per cent plant survival values were converted to arc sign values for analysis and data computation.

\section{DNA extraction and PCR}

10-15 days young leaves were collected and the DNA was extracted from resistant, mutants and susceptible checks using CTAB DNA isolation method.

DNA quality was visualized by using 0.8 per cent agarose gel electrophoresis and suitable dilutions were made for amplification.

\section{Primer amplification}

Extracted DNA was diluted as $5 \mathrm{ng}^{-1} \mu \mathrm{per}^{-1}$. The nucleotide sequence of primers is given in table 2. The markers were amplified in 25 $\mu \mathrm{l}$ reaction volume of Yeastern bio tech containing $12.5 \mathrm{ul}$ of $2 \mathrm{X}$ plant PCR mix, $1 \mu \mathrm{l}$ of YB direct DNA polymerase, $1 \mu 1$ of P1 buffer, $1 \mu \mathrm{l}$ of P2 buffer, $1.2 \mu \mathrm{l}$ of primer and $7.1 \mu \mathrm{l}$ of double distilled water. The list of primers used in the study with their thermal profile is given in table 1 .

\section{Data analysis}

Since RAPD markers are dominant, it was assumed that each band represented the phenotype at a single allelic locus (Williams et al., 1990). One molecular weight marker, 100bp DNA ladder was used to estimate the size of the amplification products. All distinct bands or fragments (RAPD markers) were thereby given identification numbers according to their position on gel and scored visually on the basis of their presence (1) or absence (0), separately for each individual and each primer.

The scores obtained using all primers in the RAPD analysis were then pooled to create a single data matrix. This was used to estimate polymorphic loci (Nei's 1973), gene diversity, genetic distance (D) and to construct a UPGMA (Unweighted Pair Group Method with Arithmetic Means) dendrogram among populations using computer program, NTSYS-PC (Version 1.31) (Spooner et al., 1996).

Pair wise genetic similarities (Sij) between genotypes were estimated by Jaccard similarity coefficient. Clustering was done using the symmetric matrix of similarity coefficient and cluster obtained based on unweighted pair group arithmetic mean (UPGMA) using NTSYS-PC.

The similarity measurements were converted to genetic distance measurements as (1-SM) X 100 (Spooner et al., 1996). Polymorphic information content values for primers were calculated following the formula (Nei, 1973).

\section{Results and Discussion}

Screening of chilli mutants against $F$. Solani

Within 5-7 days of inoculation a typical wilting symptom was noticed. Disease 
symptoms were characterized by an initial slight yellowing of the foliage and wilting of the upper leaves that progress in a few days into a permanent wilt with the leaves still attached.

On the young seedlings, initially water soaked areas developed at the collar region and a brown sunken lesion which soon appeared as girdled resulting in seedling collapse where underground stems were found dry, brown but the roots were soft and the vascular system of the lower stem was discolored and the infected plant ultimately wilted (Fig. 1).

In order to identify the resistant genotypes to Fusarium wilt, In $\mathrm{M}_{2}$ generation among 62 selected genotypes 26 genotypes showed resistant reaction viz., $\mathrm{P} 3 \mathrm{~T}_{1}-2, \mathrm{P} 3 \mathrm{~T}_{1}-6, \mathrm{P} 3$ $\mathrm{T}_{1}-8, \mathrm{P} 3 \mathrm{~T}_{1}-9, \mathrm{P} 3 \mathrm{~T}_{1}-11, \mathrm{P} 3 \mathrm{~T}_{1}-14, \mathrm{P} 3 \mathrm{~T}_{1}-15$, P3 T1-16, P3 T1-17, P3 T 1 -18, P3 T 1 -20, P3 $\mathrm{T}_{1}-24, \mathrm{P} 3 \mathrm{~T}_{1}-29$ (13 genotypes when treated with $0.5 \%$ EMS), P3 $\mathrm{T}_{2}-2, \mathrm{P} 3 \mathrm{~T}_{2}-4, \mathrm{P} 3 \mathrm{~T}_{2}-5$, P3 $\mathrm{T}_{2}-6, \mathrm{P} 3 \mathrm{~T}_{2}-7$, P3 $\mathrm{T}_{2-8}, \mathrm{P} 3 \mathrm{~T}_{2}-26$ (7 genotypes when treated with 1.0\% EMS), P3 $\mathrm{T}_{3}-1, \mathrm{P} 3 \mathrm{~T}_{3}-6, \mathrm{P} 3 \mathrm{~T}_{3}-7, \mathrm{P} 3 \mathrm{~T}_{3}-9, \mathrm{P} 3 \mathrm{~T}_{3}-12, \mathrm{P} 3$ $\mathrm{T}_{3}-14$ (6 genotypes when treated with $1.5 \%$ EMS), 32 genotypes namely $\mathrm{P} 3 \mathrm{~T}_{1}-1, \mathrm{P} 3 \mathrm{~T}_{1}-3$, P3 $\mathrm{T}_{1}-4, \mathrm{P} 3 \mathrm{~T}_{1}-5, \mathrm{P} 3 \mathrm{~T}_{1}-7, \mathrm{P} 3 \mathrm{~T}_{1}-10, \mathrm{P} 3 \mathrm{~T}_{1}-12$, P3 $\mathrm{T}_{1}-13, \mathrm{P} 3 \mathrm{~T}_{1}-19, \mathrm{P} 3 \mathrm{~T}_{1}-23, \mathrm{P} 3 \mathrm{~T}_{1}-26, \mathrm{P} 3$ $\mathrm{T}_{1}-27, \mathrm{P} 3 \mathrm{~T}_{1}-28, \mathrm{P} 3 \mathrm{~T}_{1}-31$ (15 genotypes when treated with $0.5 \%$ EMS,) P3 $\mathrm{T}_{2}-10, \mathrm{P} 3$ $\mathrm{T}_{2}-11, \mathrm{P} 3 \mathrm{~T}_{2}-13, \mathrm{P} 3 \mathrm{~T}_{2}-16, \mathrm{P} 3 \mathrm{~T}_{2}-17, \mathrm{P} 3 \mathrm{~T}_{2}-$ 18, P3 $\mathrm{T}_{2}-19, \mathrm{P} 3 \mathrm{~T}_{2}-20, \mathrm{P} 3 \mathrm{~T}_{2}-23, \mathrm{P} 3 \mathrm{~T}_{2}-25$, P3 $\mathrm{T}_{2}-27, \mathrm{P} 3 \mathrm{~T}_{2}-28$ (12 genotypes when treated with $1.0 \%$ EMS), $\mathrm{P} 3 \mathrm{~T}_{3}-2, \mathrm{P} 3 \mathrm{~T}_{3}-4, \mathrm{P} 3$ $\mathrm{T}_{3}-5, \mathrm{P} 3 \mathrm{~T}_{3}-8, \mathrm{P} 3 \mathrm{~T}_{3}-11$ (5 genotypes when treated with $1.5 \%$ EMS) were found to be moderately resistant, 3 genotypes namely $\mathrm{P} 3$ $\mathrm{T}_{1} 30$ (treated with $0.5 \%$ EMS), $\mathrm{P} 3 \mathrm{~T}_{2} 15$ and $\mathrm{P} 3 \mathrm{~T}_{2} 21$ (treated with $1.0 \%$ EMS) registered susceptible reaction, $\mathrm{P} 3 \mathrm{~T}_{1} 22$ (treated with $0.5 \%$ EMS) recorded the highly susceptible reaction.

In $\mathrm{M}_{3}$ generation among 73 screened genotypes 32 showed resistant reaction genotypes namely $\mathrm{P} 3 \mathrm{~T}_{1}-1, \mathrm{P} 3 \mathrm{~T}_{1}-3, \mathrm{P} 3 \mathrm{~T}_{1}-5$, P3 $\mathrm{T}_{1}-9, \mathrm{P} 3 \mathrm{~T}_{1}-12-10, \mathrm{P} 3 \mathrm{~T}_{1}-18, \mathrm{P} 3 \mathrm{~T}_{1}-20, \mathrm{P} 3$ $\mathrm{T}_{1}-24, \mathrm{P} 3 \mathrm{~T}_{1}-26$ (9 genotypes when treated with $0.5 \%$ EMS), P3 T2-5-4, P3 $\mathrm{T}_{2}-5-6, \mathrm{P} 3$ T2-7, P3 T 2 -8-7, P3 $\mathrm{T}_{2}$-10, P3 $\mathrm{T}_{2}-15-4, \mathrm{P} 3 \mathrm{~T}_{2}$ -16, P3 $\mathrm{T}_{2}-17, \mathrm{P} 3 \mathrm{~T}_{2}-18, \mathrm{P} 3 \mathrm{~T}_{2}-19, \mathrm{P} 3 \mathrm{~T}_{2}-$ 20-4, P3 $\mathrm{T}_{2}-20-5, \mathrm{P} 3 \mathrm{~T}_{2}-21-6, \mathrm{P} 3 \mathrm{~T}_{2}-21-7$, P3 $\mathrm{T}_{2}-23-4, \mathrm{P} 3 \mathrm{~T}_{2}-23-5, \mathrm{P} 3 \mathrm{~T}_{2}-25, \mathrm{P} 3 \mathrm{~T}_{2}-26$, P3 $\mathrm{T}_{2}-27$ (19 genotypes when treated with $1.0 \%$ EMS), $\mathrm{P} 3 \mathrm{~T}_{3}-1, \mathrm{P} 3 \mathrm{~T}_{3}-14, \mathrm{P} 3 \mathrm{~T}_{3}-11-10$, P3 $\mathrm{T}_{3}-5-2$ (4 genotypes when treated with $1.5 \%$ EMS), 29 genotypes namely $\mathrm{P} 3 \mathrm{~T}_{1}-4$, P3 $\mathrm{T}_{1}-12-3, \mathrm{P} 3 \mathrm{~T}_{1}-13, \mathrm{P} 3 \mathrm{~T}_{1}-15, \mathrm{P} 3 \mathrm{~T}_{1}-16, \mathrm{P} 3$ $\mathrm{T}_{1}$-19-2, P3 $\mathrm{T}_{1}-19-8, \mathrm{P} 3 \mathrm{~T}_{1}-22-4, \mathrm{P} 3 \mathrm{~T}_{1}-22-6$, P3 T 1 -23-2, P3 T 1 -23-4, P3 T 1 -27, P3 T 1 -29-6, P3 $\mathrm{T}_{1}-30$ (14 genotypes when treated with $0.5 \%$ EMS), P3 $\mathrm{T}_{2}-2-4, \mathrm{P} 3 \mathrm{~T}_{2}-2-9, \mathrm{P} 3 \mathrm{~T}_{2}-4$, P3 $\mathrm{T}_{2}-8-3, \mathrm{P} 3 \mathrm{~T}_{2}-6, \mathrm{P} 3 \mathrm{~T}_{2}-11$ (6 genotypes when treated with $1.0 \%$ EMS), P3 $\mathrm{T}_{3}-2-3, \mathrm{P} 3$ $\mathrm{T}_{3}-2-8, \mathrm{P} 3 \mathrm{~T}_{3}-3, \mathrm{P} 3 \mathrm{~T}_{3}-5-6, \mathrm{P} 3 \mathrm{~T}_{3}-8, \mathrm{P} 3 \mathrm{~T}_{3}-$ 9-2, P3 $\mathrm{T}_{3}-9-5, \mathrm{P} 3 \mathrm{~T}_{3}-11-3, \mathrm{P} 3 \mathrm{~T}_{3}-13$ (9 genotypes when treated with $1.5 \%$ EMS) were found to be moderately resistant, susceptible reaction was recorded in 12 genotypes namely $\mathrm{P} 3 \mathrm{~T}_{1}-6-1, \mathrm{P} 3 \mathrm{~T}_{1}-6-8, \mathrm{P} 3$ $\mathrm{T}_{1}-7, \mathrm{P} 3 \mathrm{~T}_{1}-8-7, \mathrm{P} 3 \mathrm{~T}_{1}-8-8, \mathrm{P} 3 \mathrm{~T}_{1}-10, \mathrm{P} 3 \mathrm{~T}_{1}$ -11, P3 $\mathrm{T}_{1}-28, \mathrm{P} 3 \mathrm{~T}_{1}-29-1, \mathrm{P} 3 \mathrm{~T}_{1}-3110$ genotypes (when treated with $0.5 \%$ EMS), P3 $\mathrm{T}_{2}-15-6$ when treated with $1.0 \%$ EMS, P3 $\mathrm{T}_{3-}$ 4 when treated with $1.5 \%$ EMS none of the genotypes were found to be highly susceptible (Table 2).

Such wide responses of chilli genotypes to Fusarium wilt disease was earlier observed by Ahmed et al., (1994), Singh et al., (1998), Joshi et al., (2012), Akram et al., (2014), Singh et al., (1996) and Slime and Ilhan (2010).

\section{Molecular characterization of chilli mutants}

In the present study seven Random Amplified Polymorphic DNA (RAPD) markers were used to assess the molecular diversity and characterization of chilli mutant genotypes. 
Molecular profiles were generated with RAPD primers (Figs. 2 and 3). While working with the germplasms strong and weak bands were produced in the RAPD reactions. Weak bands resulted from low homology between the primers and the pairing site on the DNA strand. The clear and unambiguous bands of seven RAPD primers were scored and used for analysis. All the seven primers showed different banding patterns and number of fragments amplified per primer varied from 2 - 6 (Table 3). The DNA polymorphisms were detected by band presence versus band absence and may be caused by failure to prime a site in some individuals due to nucleotide sequence differences or by insertions or deletions between priming sites (Clark and Langigan, 1993). Primer OPU-10 yielded maximum number of bands (6) whereas primer OPS-05 produced the least number of bands (2). The seven primers yielded a total of 31 distinct bands of which $23(74.12 \%)$ were considered as polymorphic and $8(25.80 \%)$ considered as monomorphic.

Primer OPU-10 produced highest number of polymorphic bands (6). Thus it showed higher level of polymorphism. On the other hand, OPS-05, OPU-19 and OPAP-01 primers produced lowest number of bands (2). These findings are in conformity with Paran et al., (1998) (90\% in chilli), Ashrafuzzaman (2007) (95.64\% in rice), Moonmoon (2006) (90.19\% in tomato), Joyanti (2007) (95.64\% and $88.64 \%$, respectively in large and small group cucumber) Vibha et al., (2005) $(94.36 \%)$ in rice and Gouri Sankar et al., (2014).

Table.1 Primers and their thermal profile for amplification

\begin{tabular}{|c|c|c|c|}
\hline Sl. No. & Primers & $\begin{array}{c}\text { Thermal profile } \\
\end{array}$ & No. of cycles \\
\hline 1 & $\begin{array}{l}\text { OPU-08 } \\
\text { OPU-10 } \\
\text { OPS-05 } \\
\text { OPU-03 } \\
\text { OPU-19 }\end{array}$ & $\begin{array}{l}\text { Initial denaturation }-94^{\circ} \mathrm{c} \text { for } 4 \mathrm{~min} \\
\text { Denaturation }-94^{\circ} \mathrm{c} \text { for } 1 \mathrm{~min} \\
\text { Annealing }-37^{\circ} \mathrm{c} \text { for } 2 \mathrm{~min} \\
\text { Extension }-72^{\circ} \mathrm{c} \text { for } 2 \mathrm{~min} \\
\text { Final extension }-72^{\circ} \mathrm{c} \text { for } 10 \mathrm{~min}\end{array}$ & 40 \\
\hline 2 & OPD-20 & $\begin{array}{l}\text { Initial denaturation }-94^{\circ} \mathrm{c} \text { for } 4 \mathrm{~min} \\
\text { Denaturation }-94^{\circ} \mathrm{c} \text { for } 1 \mathrm{~min} \\
\text { Annealing }-35.4^{\circ} \mathrm{c} \text { for } 1 \mathrm{~min} \\
\text { Extension }-72^{\circ} \mathrm{c} \text { for } 1 \mathrm{~min} \\
\text { Final extension }-72^{\circ} \mathrm{c} \text { for } 10 \mathrm{~min}\end{array}$ & 30 \\
\hline 3 & OPAP-01 & $\begin{array}{l}\text { Initial denaturation }-93^{\circ} \mathrm{c} \text { for } 2 \mathrm{~min} \\
\text { Denaturation }-93^{\circ} \mathrm{c} \text { for } 30 \mathrm{sec} \\
\text { Annealing }-35^{\circ} \mathrm{c} \text { for } 30 \mathrm{sec} \\
\text { Extension }-72^{\circ} \mathrm{c} \text { for } 90 \mathrm{sec} \\
\text { Final extension }-72^{\circ} \mathrm{c} \text { for } 5 \mathrm{~min}\end{array}$ & 40 \\
\hline
\end{tabular}

$\mathrm{n}$

$\operatorname{PIC} \mathrm{j}=1-\Sigma 1-\mathrm{Pij} 2$

$\mathrm{i}=1$

Where,

$\mathrm{Pi}$ is the frequency for the jth allele for marker $\mathrm{i}$ and summation extends over $\mathrm{n}$ alleles. 
Int.J.Curr.Microbiol.App.Sci (2017) 6(9): 1585-1597

Table.2 Reaction of $\mathrm{M}_{2}$ and $\mathrm{M}_{3}$ chilli mutant genotypes against Fusarium wilt

\begin{tabular}{|c|c|c|c|c|c|c|c|}
\hline \multirow{2}{*}{$\begin{array}{l}\text { Sl. } \\
\text { No. }\end{array}$} & \multirow{2}{*}{$\begin{array}{l}\% \text { Disease } \\
\text { reaction }\end{array}$} & \multirow{2}{*}{$\begin{array}{c}\text { EMS } \\
\text { Treatment } \\
\text { Doses }\end{array}$} & \multicolumn{2}{|c|}{ Genotypes } & \multicolumn{2}{|c|}{ Total } & \multirow[t]{2}{*}{ Remarks } \\
\hline & & & $\mathrm{M}_{2}$ Generation & $\mathrm{M}_{3}$ Generation & $\begin{array}{c}M_{2} \\
\text { Generation }\end{array}$ & $\begin{array}{c}M_{3} \\
\text { Generation }\end{array}$ & \\
\hline \multirow{3}{*}{1.} & \multirow{3}{*}{$>75 \%$} & $0.5 \%$ & $\begin{array}{l}\text { P3 } \mathrm{T}_{1} 2, \mathrm{P} 3 \mathrm{~T}_{1} 6, \mathrm{P} 3 \mathrm{~T}_{1} 8, \mathrm{P} 3 \mathrm{~T}_{1} \text { 9, } \mathrm{P} 3 \mathrm{~T}_{1} 11, \\
\mathrm{P} 3 \mathrm{~T}_{1} 14, \mathrm{P} 3 \mathrm{~T}_{1} 15, \mathrm{P} 3 \mathrm{~T}_{1} 16, \mathrm{P} 3 \mathrm{~T}_{1} 17, \mathrm{P} 3 \mathrm{~T}_{1} \\
\text { 18, } \mathrm{P} 3 \mathrm{~T}_{1} 20, \mathrm{P} 3 \mathrm{~T}_{1} 24, \mathrm{P} 3 \mathrm{~T}_{1} 29\end{array}$ & $\begin{array}{l}\mathrm{P} 3 \mathrm{~T}_{1} 1, \mathrm{P} 3 \mathrm{~T}_{1} 3, \mathrm{P} 3 \mathrm{~T}_{1} 5, \mathrm{P} 3 \mathrm{~T}_{1} \text { 9, } \mathrm{P} 3 \mathrm{~T}_{1} 12-10, \mathrm{P} 3 \\
\mathrm{~T}_{1} 18, \mathrm{P} 3 \mathrm{~T}_{1} 20, \mathrm{P} 3 \mathrm{~T}_{1} 24, \mathrm{P} 3 \mathrm{~T}_{1} 26\end{array}$ & \multirow{3}{*}{26} & \multirow{3}{*}{32} & \multirow{3}{*}{ Resistant } \\
\hline & & $1.0 \%$ & $\begin{array}{l}\mathrm{P} 3 \mathrm{~T}_{2} 2, \mathrm{P} 3 \mathrm{~T}_{2} 4, \mathrm{P} 3 \mathrm{~T}_{2} 5, \mathrm{P} 3 \mathrm{~T}_{2} 6, \mathrm{P} 3 \mathrm{~T}_{2} 7 \\
\mathrm{P} 3 \mathrm{~T}_{2} 8, \mathrm{P} 3 \mathrm{~T}_{2} 26\end{array}$ & $\begin{array}{l}\mathrm{P} 3 \mathrm{~T}_{2} \text { 5-4, P3 } \mathrm{T}_{2} \text { 5-6, P3 } \mathrm{T}_{2} \text { 7, P3 } \mathrm{T}_{2} 8-7, \mathrm{P} 3 \mathrm{~T}_{2} 10, \mathrm{P} 3 \\
\mathrm{~T}_{2} \text { 15-4, P3 } \mathrm{T}_{2} 16, \mathrm{P} 3 \mathrm{~T}_{2} 17, \mathrm{P} 3 \mathrm{~T}_{2} 18, \mathrm{P} 3 \mathrm{~T}_{2} 19, \mathrm{P} 3 \\
\mathrm{~T}_{2} \text { 20-4, P3 } \mathrm{T}_{2} \text { 20-5, P3 } \mathrm{T}_{2} 21-6, \mathrm{P} 3 \mathrm{~T}_{2} 21-7, \mathrm{P} 3 \mathrm{~T}_{2} \\
\text { 23-4, P3 } \mathrm{T}_{2} 23-5, \mathrm{P} 3 \mathrm{~T}_{2} 25, \mathrm{P} 3 \mathrm{~T}_{2} 26, \mathrm{P} 3 \mathrm{~T}_{2} 27\end{array}$ & & & \\
\hline & & $1.5 \%$ & $\begin{array}{l}\mathrm{P} 3 \mathrm{~T}_{3} 1, \mathrm{P} 3 \mathrm{~T}_{3} 6, \mathrm{P} 3 \mathrm{~T}_{3} 7, \mathrm{P} 3 \mathrm{~T}_{3} 9, \mathrm{P} 3 \mathrm{~T}_{3} 12, \\
\mathrm{P} 3 \mathrm{~T}_{3} 14\end{array}$ & $\mathrm{P} 3 \mathrm{~T}_{3} 1, \mathrm{P} 3 \mathrm{~T}_{3} 14, \mathrm{P} 3 \mathrm{~T}_{3} 11-10, \mathrm{P} 3 \mathrm{~T}_{3} 5-2$ & & & \\
\hline \multirow{3}{*}{2.} & \multirow{3}{*}{$50-75 \%$} & $0.5 \%$ & $\begin{array}{l}\text { P3 } \mathrm{T}_{1} 1, \mathrm{P} 3 \mathrm{~T}_{1} 3, \mathrm{P} 3 \mathrm{~T}_{1} 4, \mathrm{P} 3 \mathrm{~T}_{1} 5, \mathrm{P} 3 \mathrm{~T}_{1} 7, \\
\mathrm{P} 3 \mathrm{~T}_{1} \text { 10, } \mathrm{P} 3 \mathrm{~T}_{1} 12, \mathrm{P} 3 \mathrm{~T}_{1} 13, \mathrm{P} 3 \mathrm{~T}_{1} 19, \mathrm{P} 3 \mathrm{~T}_{1} \\
\text { 23, P3 } \mathrm{T}_{1} 26, \mathrm{P} 3 \mathrm{~T}_{1} 27, \mathrm{P} 3 \mathrm{~T}_{1} 28, \mathrm{P} 3 \mathrm{~T}_{1} 31\end{array}$ & 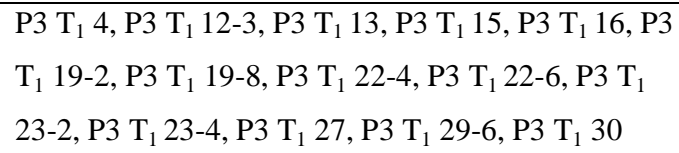 & \multirow{3}{*}{32} & \multirow{3}{*}{29} & \multirow{3}{*}{$\begin{array}{c}\text { Moderately } \\
\text { resistant }\end{array}$} \\
\hline & & $1.0 \%$ & $\begin{array}{l}\text { P3 } \mathrm{T}_{2} 10, \mathrm{P} 3 \mathrm{~T}_{2} 11, \mathrm{P} 3 \mathrm{~T}_{2} 13, \mathrm{P} 3 \mathrm{~T}_{2} 16, \mathrm{P} 3 \mathrm{~T}_{2} \\
\text { 17, P3 } \mathrm{T}_{2} 18, \mathrm{P} 3 \mathrm{~T}_{2} 19, \mathrm{P} 3 \mathrm{~T}_{2} 20, \mathrm{P} 3 \mathrm{~T}_{2} 23, \\
\text { P3 } \mathrm{T}_{2} 25, \mathrm{P} 3 \mathrm{~T}_{2} 27, \mathrm{P} 3 \mathrm{~T}_{2} 28\end{array}$ & $\begin{array}{l}\mathrm{P} 3 \mathrm{~T}_{2} 2-4, \mathrm{P} 3 \mathrm{~T}_{2} 2-9, \mathrm{P} 3 \mathrm{~T}_{2} 4, \mathrm{P} 3 \mathrm{~T}_{2} 8-3, \mathrm{P} 3 \mathrm{~T}_{2} 6, \mathrm{P} 3 \\
\mathrm{~T}_{2} 11\end{array}$ & & & \\
\hline & & $1.5 \%$ & $\mathrm{P} 3 \mathrm{~T}_{3} 2, \mathrm{P} 3 \mathrm{~T}_{3} 4, \mathrm{P} 3 \mathrm{~T}_{3} 5, \mathrm{P} 3 \mathrm{~T}_{3} 8, \mathrm{P} 3 \mathrm{~T}_{3} 11$ & $\begin{array}{l}\mathrm{P} 3 \mathrm{~T}_{3} 2-3, \mathrm{P} 3 \mathrm{~T}_{3} 2-8, \mathrm{P} 3 \mathrm{~T}_{3} 3, \mathrm{P} 3 \mathrm{~T}_{3} 5-6, \mathrm{P} 3 \mathrm{~T}_{3} 8, \mathrm{P} 3 \\
\mathrm{~T}_{3} 9-2, \mathrm{P} 3 \mathrm{~T}_{3} \text { 9-5, P3 } \mathrm{T}_{3} \text { 11-3, P3 } \mathrm{T}_{3} 13\end{array}$ & & & \\
\hline \multirow{3}{*}{3.} & \multirow{3}{*}{$25-50 \%$} & $0.5 \%$ & $\mathrm{P} 3 \mathrm{~T}_{1} 30$ & $\begin{array}{l}\text { P3 } \mathrm{T}_{1} 6-1, \mathrm{P} 3 \mathrm{~T}_{1} 6-8, \mathrm{P} 3 \mathrm{~T}_{1} \text { 7, } \mathrm{P} 3 \mathrm{~T}_{1} 8-7, \mathrm{P} 3 \mathrm{~T}_{1} 8-8, \\
\mathrm{P} 3 \mathrm{~T}_{1} 10, \mathrm{P} 3 \mathrm{~T}_{1} 11, \mathrm{P} 3 \mathrm{~T}_{1} 28, \mathrm{P} 3 \mathrm{~T}_{1} 29-1, \mathrm{P} 3 \mathrm{~T}_{1} 31\end{array}$ & \multirow{3}{*}{3} & \multirow{3}{*}{12} & \multirow{3}{*}{ Susceptible } \\
\hline & & $1.0 \%$ & $\mathrm{P} 3 \mathrm{~T}_{2} 15, \mathrm{P} 3 \mathrm{~T}_{2} 21$ & $\mathrm{P} 3 \mathrm{~T}_{2} 15-6$ & & & \\
\hline & & $1.5 \%$ & ---- & $\mathrm{P} 3 \mathrm{~T}_{3} 4$ & & & \\
\hline \multirow{2}{*}{4.} & \multirow[t]{2}{*}{$0-25 \%$} & $0.5 \%$ & $\mathrm{P} 3 \mathrm{~T}_{1} 22$ & ------ & \multirow{2}{*}{1} & \multirow{2}{*}{0} & \multirow{2}{*}{$\begin{array}{c}\text { Highly } \\
\text { susceptible }\end{array}$} \\
\hline & & $1.0 \%$ & -------- & -------- & & & \\
\hline
\end{tabular}

EMS - Ethyl Methyl Sulphonate 
Table.3 RAPD banding profile of different primers for chilli mutants

\begin{tabular}{|c|c|c|c|c|}
\hline Sl. No. & Primers & Total No. of bands & No. of polymorphic bands & Polymorphism (\%) \\
\hline 1 & OPU-08 & 5 & 3 & 60 \\
\hline 2 & OPU-10 & 6 & 6 & 100 \\
\hline 3 & OPS-05 & 2 & 2 & 100 \\
\hline 4 & OPU-03 & 5 & 5 & 100 \\
\hline 5 & OPU-19 & 4 & 2 & 50 \\
\hline 6 & OPD-20 & 4 & 3 & 75 \\
\hline 7 & OPAP-01 & 5 & 2 & 40 \\
\hline & Total & 31 & 23 & 525 \\
\cline { 2 - 5 } & Average & 4.4 & 3.29 & 75 \\
\hline
\end{tabular}

Table.4 Unique bands obtained in the RAPD profile

\begin{tabular}{|c|c|c|c|c|c|c|c|}
\hline Sl. No. & Primers & P $_{\mathbf{3}}$ & B.D & US & R1 & S & R2 \\
\hline 1 & OPU-08 & 0 & 1 & 0 & 0 & 0 & 0 \\
\hline 2 & OPU-10 & 0 & 0 & 0 & 0 & 0 & 0 \\
\hline 3 & OPS-05 & 0 & 0 & 0 & 0 & 0 & 0 \\
\hline 4 & OPU-03 & 0 & 0 & 1 & 0 & 0 & 0 \\
\hline 5 & OPU-19 & 1 & 1 & 0 & 1 & 0 & 0 \\
\hline 6 & OPD-20 & 0 & 1 & 0 & 0 & 0 & 0 \\
\hline 7 & OPAP-01 & 0 & 1 & 0 & 0 & 0 & 0 \\
\hline
\end{tabular}

Table.5 Similarity matrix of chilli mutant genotypes based on RAPD profiles

\begin{tabular}{|c|c|c|c|c|c|c|}
\hline Genotype & P3 & B.D & US & R1 & S & R2 \\
\hline P3 & $\mathbf{1 . 0 0}$ & & & & & \\
\hline BD & 0.74 & $\mathbf{1 . 0 0}$ & & & & \\
\hline US & 0.71 & 0.78 & $\mathbf{1 . 0 0}$ & & & \\
\hline R1 & 0.68 & 0.72 & 0.73 & $\mathbf{1 . 0 0}$ & & \\
\hline S & 0.74 & 0.82 & 0.74 & 0.67 & $\mathbf{1 . 0 0}$ & \\
\hline R2 & 0.73 & 0.76 & 0.82 & 0.90 & 0.76 & $\mathbf{1 . 0 0}$ \\
\hline
\end{tabular}

P3- Resistant check

R1- Resistant bulk DNA
B.D- Susceptible check US- Farmers variety

S- Susceptible bulk DNA R2- Resistant bulk DNA

Cluster analysis

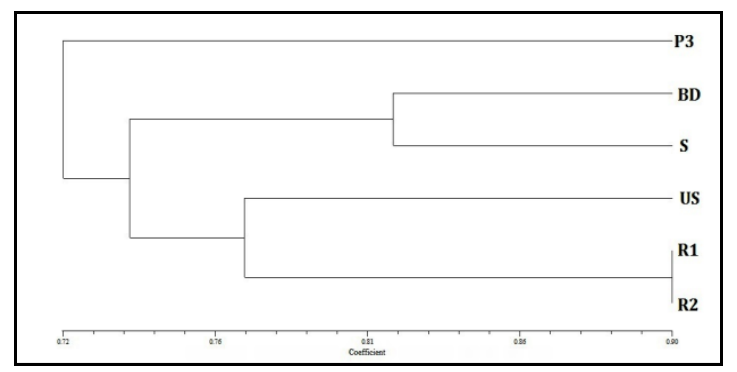

P3- Resistant check

B.D- Susceptible check S- Susceptible bulk DNA

US- Farmers variety

R1- Resistant bulk DNA R2- Resistant bulk DNA 
Fig.1 Rapid root dip transplanting technique for the screening of Chilli mutants for its resistance to $F$. solani
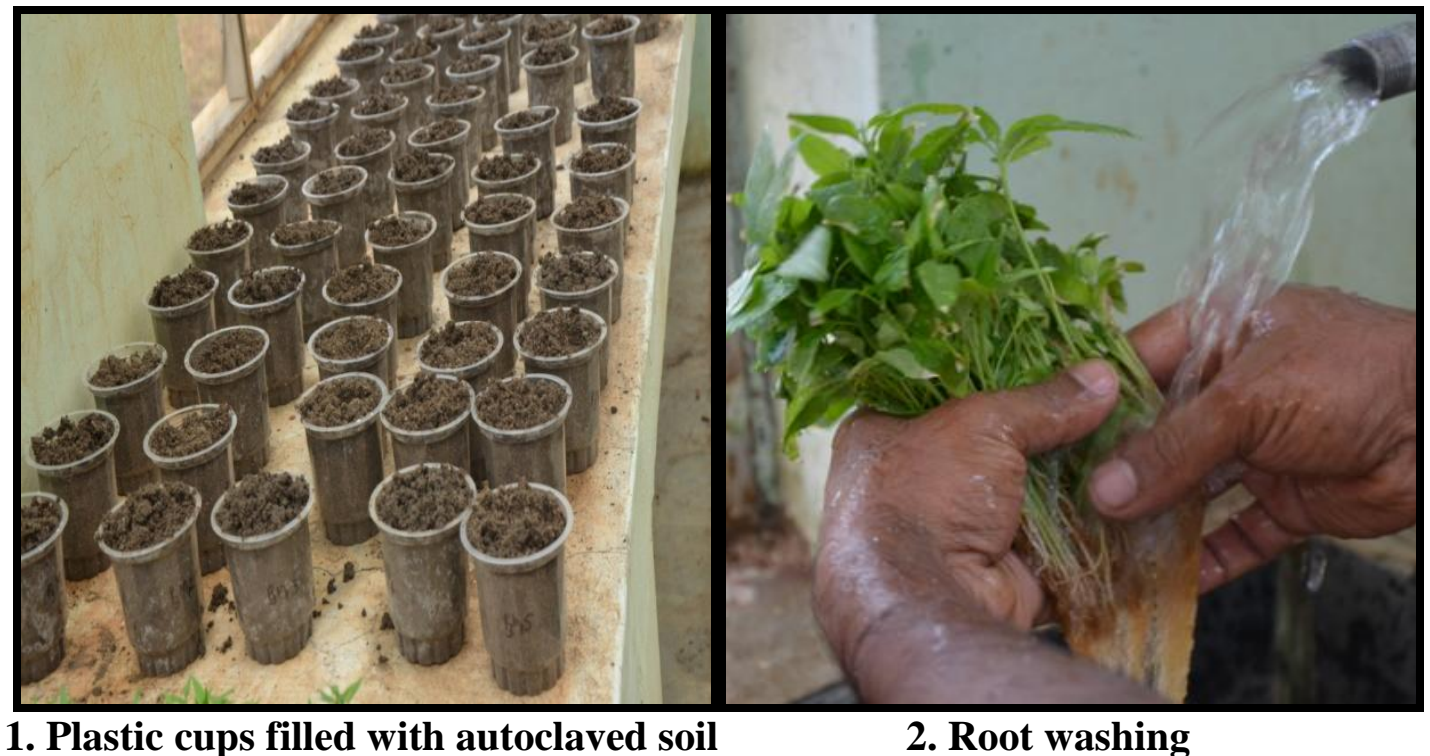

2. Root washing

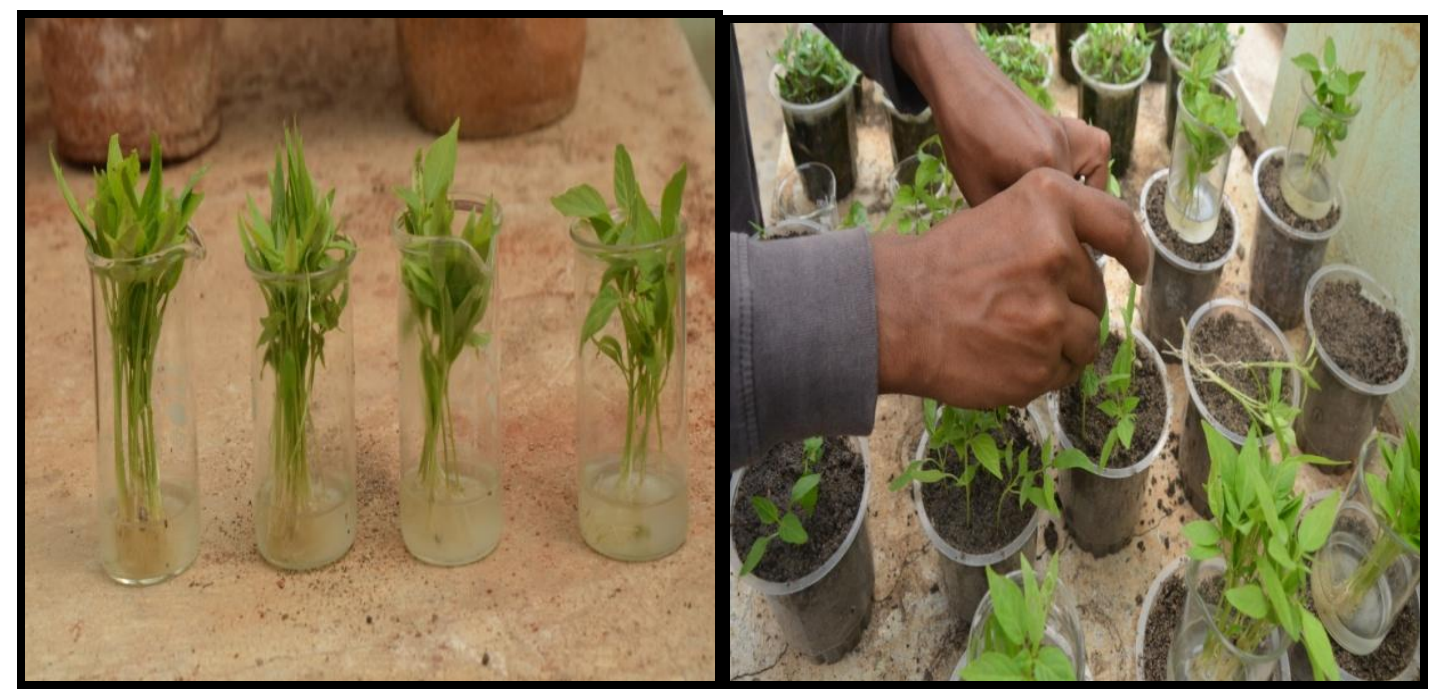

3.Root dipping in F.solani inoculum 4.Transplanting of pre inoculated seedlings

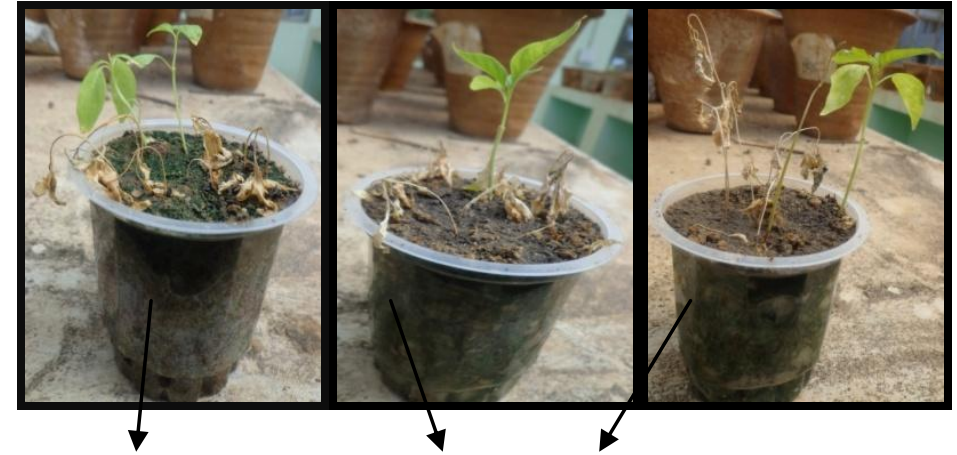

5.Susceptible genotypes showing wilting symptom

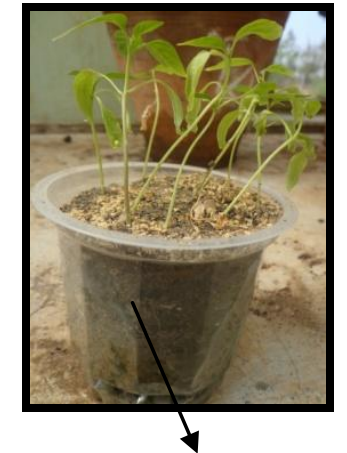

6.Resistant genotypes 
Fig.2 RAPD profiles of Chilli genotypes and mutants generated by the primers OPU-08, OPU-05, OPS-10, OPU-03 and OPU-19
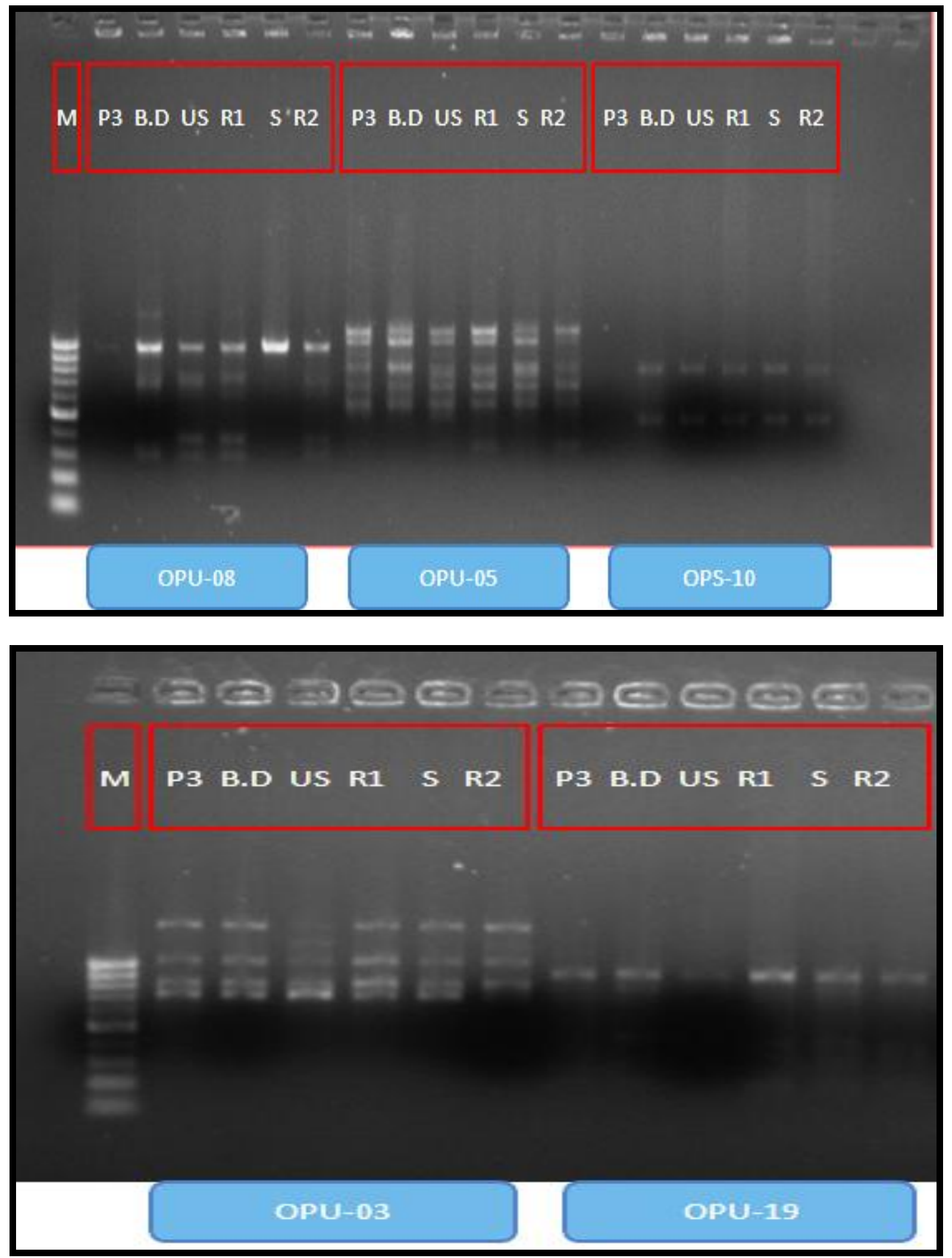
Fig.3 RAPD profiles of Chilli genotypes and mutants generated by the primers OPD-20 and OPAP-01
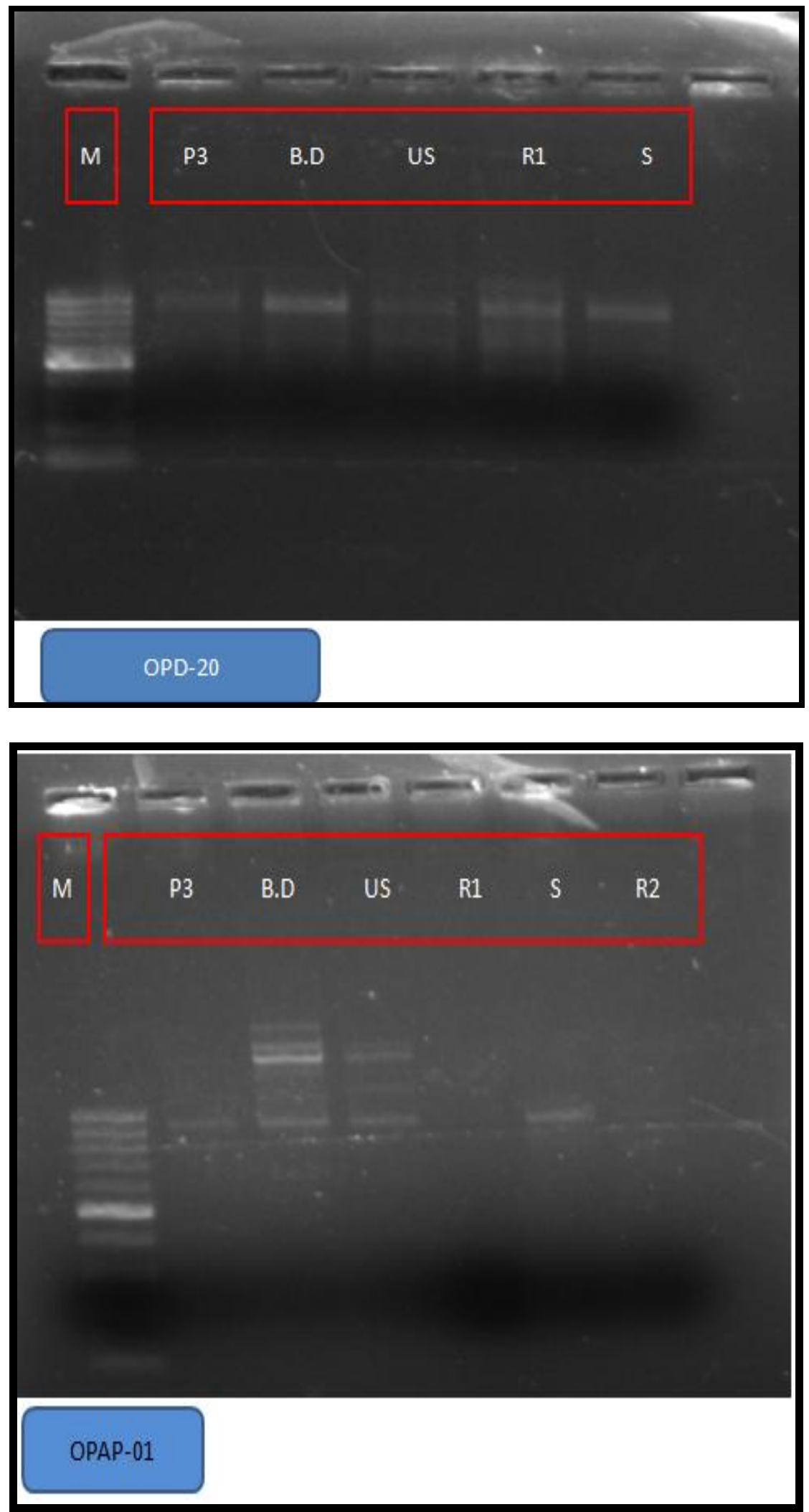


\section{Unique bands obtained in RAPD profile}

The unique bands obtained in the present study were 8 in number (Table 4) and this allows us to differentiate resistance and susceptible plants and further could be used for developing SCAR markers for evaluating or differentiating resistant and susceptible genotypes.

\section{Similarity matrix analysis using molecular data}

Based on Jaccard's coefficient method genetic similarity matrix was constructed using RAPD marker data to assess the genetic relatedness among all the genotypes. Genetic similarity of 0.9 was found between the genotype R1 $\left(1^{\text {st }}\right.$ Resistant pool of mutants $)$ and R2 ( $2^{\text {nd }}$ Resistant pool of mutants) indicating their close relationship at molecular level as they belonged to the same cluster. The lowest genetic similarity of 0.67 was found between R1 ( $1^{\text {st }}$ Resistant pool of mutants) and $\mathrm{S}$ (Susceptible pool of mutants) and 0.76 between R2 ( $2^{\text {nd }}$ Resistant pool of mutants) and S (Susceptible pool of mutants) revealing the high divergence among these genotypes (Table 5). In these genotypes the genomic region may be similar or different depends upon genetic makeup of an individual and crossing among different groups of genotypes would be desirable for breeders, these findings are in similarity with Paran et al., (1998) and Singh et al., (2013).

\section{Cluster analysis}

72 mutant genotypes were grouped into three clusters at 0.78 Jaccard's similarity coefficient (Fig. 4). Cluster I was the smallest comprising of only one genotype which was resistant check (P3). Cluster II had two genotypes of which both were susceptible namely a susceptible check (B.dabbi) and susceptible pool of mutants and III had 3 genotypes all of which were resistant, among them there were two resistance pool of mutants and a local genotype namely US-341 which were also found resistant in screening of genotypes, indicating high degree of similarity between these genotypes.

This study noticed that considerable amount of molecular diversity exists in chilli genotypes. Present result found similar to Pervaiz (2006) in rice crop and Singh et al., (2013) in pigeon pea.

Based on present findings the resistant genotypes can be further used for selecting and stabilizing suitable genotypes against Fusarium wilt and resistance breeding programme in chilli Capsicum annuum $\mathrm{L}$. Polymorphic information content ranged from $40 \%$ to $100 \%$. The primers OPU-08, OPS- 10 and OPAP-1 were useful in molecular characterization for Fusarium wilt screening. Similarly OPU-08 primer produced specific band for susceptible line and for resistant line oligo OPU-19 could be a better distinguishing. Further studies are carried out to develop SCAR markers using findings from the present investigation. This will be suitable for Fusarium wilt resistance source screening for the Breeders and Biotechnologists.

\section{Acknowledgments}

B. V. Tembhurne, B. Kisan, D. S. Ashwathanarayana, Nidagundi: Conceived theme of the study.

B. V. Tembhurne, B. Belabadevi B. Kisan, D. S. Ashwathanarayana, Suvarna: Conducted experiment, Data analysis.

B. V. Tembhurne, B. Kisan, Tilak I.S, and M. K. Naik: Drafted the manuscript.

B. V. Tembhurne, B. Kisan, B. Belabadevi, D. S. Ashwathanarayana, Tilak I.S: Edited the manuscript. 
All authors read and approved the final manuscript

Department: Genetics and plant breeding.

University: University of Agricultural Sciences Raichur.

\section{References}

Ahmed, N., Tanki, M. I. and Mir, N. M., 1994. Screening of advance breeding lines of chilli and sweet and hot pepper cultivars against Fusarium wilt. Pl. Dis. Res., 9(2): 153154.

Akram, W., Tehmina, A. and Aqeel, A., 2014. Basal susceptibility of tomato varieties against different isolates of Fusarium oxysporum f. sp. lycopersici. Int. J. Agric. Biol., 161: 171-176.

Anonymous, 2005. Annual report, ICAR, New Delhi. pp. 7.

Ashrafuzzaman, M., 2007. Genetic diversity analysis of rice genotypes as revealed by RAPD markers. MS thesis. Dept. of Biotechnology. Bangladesh Agricultural University, Mymensingh, Pp. 16-50.

Clark, A.G., and Langigan, C. M. S., 1993, Prospects for estimating nucleotide divergence with RAPDs, Mol. Evol. 10: 1096-1111.

Gouri Sankar, T., Gopal, K., Gopi, V. and Sreenivasulu, Y., 2014. Molecular characterization of Fusarium solani isolates causing dry root rot of sweet orange Citrus sinensis osbeck. Int. J. Curr. Microbiol. App. Sci., 33: 105-114.

Joshi, M., Srivastava, R., Sharma, A. and Anil, P., 2012 Screening of resistant varieties and antagonistic Fusarium oxysporum for biocontrol of Fusarium wilt of chilli. J. Pl. Pathol. Microbiol, 35: 134.

Joyanti, 2007 Assessment of genetic diversity and relationship among some commercial cucumber varieties and genotypes using RAPD Markers., J. Bio. Sci., Chittagong University, 6(1 \& 2): 51-63.

Konzak, C. F., Nilan, R. A. and Kleinhofs, A., 1977 Artificial mutagenesis as an aid in overcoming genetic vulnerability of crop plants. Plenum Publishing Corporation., New York, pp. 163 - 177.

Madhukar, H. M., and Naik, M. K., 2004 Evaluation of bioagents against Fusarium wilt of chilli Capsicum annuum. In: Proc. $15^{\text {th }}$ International Plant Protection Towards $21^{\text {st }}$ Century held in Beijing, China. pp. 540.

Moonmoon, S., 2006. Random amplified polymorphic DNA markers RAPD for genetic variation study among tomato varieties. MS thesis. Dept. of Biotechnology. Bangladesh Agricultural University, Mymensingh, Pp. 34-51.

Naik, M. K., Pramanick, K., Deshpande, A. H. and Sinha, Poonam, 1996. Standardization of screening technique against Fusarium wilt of chilli. National Symposium of Indian Society Mycol. Pl. Pathol. Held at Shantiniketan, West Bengal. Pp. 113-114.

Nei, M., 1973. Analysis of gene diversity in subdivided populations. Proc. Natl. Acad. Sci. USA, 70: 3321-3323.

Paran, I., Aftergoot, E. and Shifriss, C, 1998. Variation in Capsicum annuum revealed by RA-PD and AFLP markers. Euphytica, 99: 167-173.

Pervaiz, Z. H., Rabbani, A. M., Khaliq, I., Pearce, S. R. and Malik, S. A., 2006. Genetic diversity associated with agronomic traits using microsatellite markers in Pakistani rice landraces. Electron J. Pl. Breed., 13 3: 1-10.

Raghuvanshi, R. K., and D. Singh, 1982. Induced mutagenesis in Capsicum. II. Effects of single and combined mutagenic treatments on habit and fruit. Curr. Sci., 51: $235-237$. 
Rostaino, F., 1983, Pepper brachytic forms Capsicum annuum L. obtained through induced mutagenesis. Genet. Agrar, 37: $204-205$.

Sigurbjornsson, B., 1977. Induction of mutation in wheat, Academy of Sciences, Ukranian SSR, Kiev, USSR, 41: 204-217.

Silme, R. S., and Ilhan, M. C., 2010. Screening for resistance to Fusarium wilt in induced mutants and world collection of sesame under intensive management. Turkish J. Field Crops, 151: 89-93.

Singh, A., Singh, A. K. and Singh, A., 1998. Screening of chilli germplasms against Fusarium wilt. Crop Res., 15: 132-133.

Singh, A.K., Rai, V. P., Chand, R., Singh, R. P. and Singh, M. N., 2013. Genetic diversity studies and identification of SSR markers associated with Fusarium wilt Fusarium udum resistance in cultivated pigeonpea Cajanus cajan. $J$. Genet., 92: 273-280.

Singh, R.P., Harichand and Chand, H., 1996.
Identification of multiple disease resistance in chickpea at Hissar, Haryana. Int. Chickpea Pigeonpea Newslett, 3: 32-33.

Spooner, D.M., Tivang, J., Nichvis, J., Miller, J. T., Douches, D. S. and Contreras, M. A., 1996. NTSYS-PC for diversity analysis. Theor. Appl. Genet., 92: 532540.

Subhash, K., Rajam, M. V., Sathyanarayana, B. and Meerabai. A., 1981 Induction of multilocular ovary in Capsicum annuum L. by mitomycin C. Indian J. Exp. Biol., 19: $301-302$.

Vibha, G., 2005. Molecular characterization and genetic diversity sudy in F3 population of rice. Progressive Agriculture. $201 \& 2: 1-8$.

Williams, J. G. K., Kubelik, A. R., Livak, K. J., Rafalski, J. A. and Tingey, S. V., 1990. DNA polymorphism amplified by arbitrary primers are useful as genetic markers. Nucl. Acid Res., 18: 65316535.

\section{How to cite this article:}

Tembhurne, B.V., B. Belabadevi, B. Kisan, I.S. Tilak, D.S. Ashwathanarayana, Suvarna, Nidagundi and Naik, M.K. 2017. Molecular Characterization and Screening for Fusarium (Fusarium solani) Resistance in Chilli (Capsicum annuum L.) Genotypes. Int.J.Curr.Microbiol.App.Sci. 6(9): 1585-1597. doi: https://doi.org/10.20546/ijcmas.2017.609.195 